\title{
IS GAUSS QUADRATURE BETTER THAN CLENSHAW-CURTIS?
}

\author{
LLOYD N. TREFETHEN*
}

\begin{abstract}
We consider the question of whether Gauss quadrature, which is very famous, is more powerful than the much simpler Clenshaw-Curtis quadrature, which is less well-known. Sevenline MATLAB codes are presented that implement both methods, and experiments show that the supposed factor-of- 2 advantage of Gauss quadrature is rarely realized. Theorems are given to explain this effect. First, following Elliott and O'Hara and Smith in the 1960s, the phenomenon is explained as a consequence of aliasing of coefficients in Chebyshev expansions. Then another explanation is offered based on the interpretation of a quadrature formula as a rational approximation of $\log ((z+1) /(z-1))$ in the complex plane. Gauss quadrature corresponds to Padé approximation at $z=\infty$. ClenshawCurtis quadrature corresponds to an approximation whose order of accuracy at $z=\infty$ is only half as high, but which is nevertheless equally accurate near $[-1,1]$.
\end{abstract}

Key words. Newton-Cotes quadrature, Gauss quadrature, Clenshaw-Curtis quadrature, Chebyshev expansion, rational approximation, FFT, spectral methods

AMS subject classifications. 65D32, 41A20, 41A58

1. Introduction. Every numerical analysis textbook has a chapter on numerical integration. Most of these present two families of $(n+1)$-point quadrature rules: first Newton-Cotes formulas, based on equally spaced points, and then Gauss formulas, based on optimal (in a sense) points. Among the books that fit this pattern are

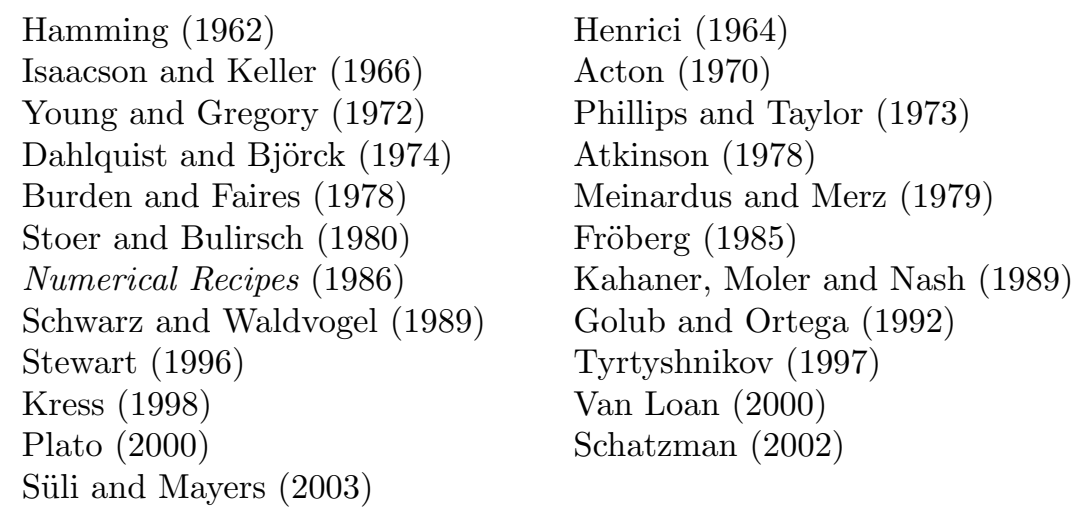

Now in fact, as the books to varying degrees make clear, Newton-Cotes formulas are useless for high values of $n$, suffering from the catastrophic $O\left(2^{n}\right)$ Runge phenomenon: they can integrate only functions that are analytic in a large region in the complex plane, and even these only in the absence of rounding errors [13,36, 37]. Gauss quadrature formulas, by contrast, have perfect robustness as well as their well-known apparent factor-of-2 advantage in efficiency: whereas the $(n+1)$-point Newton-Cotes formula exactly integrates polynomials of degree $n$, the $(n+1)$-point Gauss formula exactly integrates polynomials of degree $2 n+1$. Gauss quadrature nodes and weights take more work to calculate, but this can be done with excellent accuracy in $O\left(n^{3}\right)$ operations by solving a tridiagonal eigenvalue problem, as shown by Golub and Welsch [22]. In the end many of us absorb a simple lesson: for large $n$, Gauss quadrature is the only serious competitor.

\footnotetext{
*Oxford Computing Laboratory, Wolfson Bldg., Parks Rd., Oxford OX1 3QD, UK (LNT@comlab.ox.ac.uk).
} 
This view is mistaken, for Newton-Cotes is a straw man. In fact, we should compare Gauss quadrature with Clenshaw-Curtis quadrature, a much simpler technique based on sampling the integrand at Chebyshev points. This method also integrates polynomials of degree $n$ exactly, with no difficulties as $n \rightarrow \infty$. Clenshaw-Curtis formulas are mentioned in the textbooks of Johnson and Riess (1982), Ueberhuber (1997), Neumaier (2001), and Heath (2002), as well as more briefly in endnotes or exercises in Gautschi (1997) and Cheney and Kincaid (1999). They are not mentioned in Krylov's 1962 book on numerical integration [30], but they are treated in later books in this field including Davis and Rabinowitz (1967, 1975 and 1984), Engels (1980), and Krommer and Ueberhuber (1998) [10, 13, 14, 29]. Concerning Gauss quadrature, an indispensable source is the masterful 1981 survey by Gautschi [20].

Perhaps the following is a reasonable summary of the conventional wisdom, among the minority who are aware of Clenshaw-Curtis quadrature, of $n$-point quadrature formulas. Gauss quadrature is the gold standard, with perfect convergence and stability properties, though in some applications one might choose to rely instead on simpler formulas enhanced by adaptivity or extrapolation. Clenshaw-Curtis is a footnote, for it is only half as efficient.

We shall see that in fact, for many integrands, Clenshaw-Curtis and Gauss quadrature are equally efficient.

2. Gauss and Clenshaw-Curtis formulas. Throughout this article we are concerned with what are known as interpolatory quadrature formulas. A continuous function $f$ on $[-1,1]$ is given, and we seek to approximate the integral

$$
I=I(f)=\int_{-1}^{1} f(x) d x
$$

by sums

$$
I_{n}=I_{n}(f)=\sum_{k=0}^{n} w_{k} f\left(x_{k}\right)
$$

for various $n$, where the nodes $\left\{x_{k}\right\}$ depend on $n$ but not $f$. The weights $\left\{w_{k}\right\}$ are defined uniquely by the property that $I_{n}$ is equal to the integral of the degree $\leq n$ polynomial interpolant through the data points. Thus by construction, (2.2) gets the exactly correct answer if $f$ is a polynomial of degree $\leq n$.

Newton-Cotes formulas are defined by taking the nodes to be equally spaced from -1 to 1 . The Newton-Cotes formulas of low order are important as building blocks for discretizations of differential equations and other numerical methods. Their properties as $n \rightarrow \infty$ are very bad, however, and we shall not discuss them further except for brief reappearances in Figures 2.1 and 6.1.

Gauss formulas are defined by choosing the nodes optimally in the sense of maximizing the degree of polynomials that (2.2) integrates exactly. Since there are $n+1$ nodes, it is not surprising that the attainable degree is $n+1$ orders higher than in the generic case, i.e., degree $2 n+1$. Gauss quadrature formulas were discovered by Gauss in his mid-thirties [19], and it is a measure of the extraordinary productivity of Gauss's career that this great discovery merits hardly a mention in many accounts of his life. For these formulas are truly powerful. Their impact was mainly theoretical before the advent of computers, because of the difficulty of determining the nodes and weights and also of evaluating integrands at irrational arguments, but in the computer 
era, especially since the appearance of a paper by Golub and Welsch in 1969 (following earlier work of Goertzel 1954, Wilf 1962 and Gordon 1968), it has been practical as well [22]. We shall not give details but just offer the following MATLAB function gauss.m, which is almost the same as the code by the same name in [41].

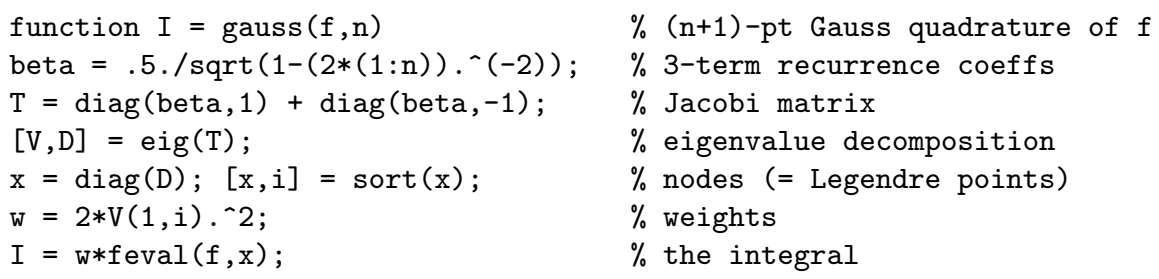

For example, the command gauss (@cos,6) yields 1.68294196961579, which is correct to full precision. The command gauss (@cos, 1000) yields the same result, though it takes 17 seconds on my laptop. ${ }^{1}$

The idea of Clenshaw-Curtis quadrature is to use Chebyshev points instead of optimal nodes. There are three main variations on this theme (see [34] for others):

Chebyshev roots in $(-1,1)$ : Fejer's "first rule" (1933) [15]

Chebyshev extrema in $(-1,1)$ : Fejer's "second rule" (1933) [15]

Chebyshev extrema in $[-1,1]$ : Clenshaw and Curtis (1960) [7]

The first and the third variants are also called "classical" and "practical" ClenshawCurtis formulas, respectively. (A number of the relevant papers published especially in the 1960s make no reference to Fejér.) It seems that the last choice may be the most accurate [12] as well as having the easiest connection with the fast Fourier transform, ${ }^{2}$ and also the potential advantage of convenient reuse of nodes when $n$ is doubled; it is certainly the one used mainly in practice. The nodes in question, which we shall simply call Chebyshev points, are defined by

$$
x_{j}=\cos \frac{j \pi}{n}, \quad 0 \leq j \leq n .
$$

The Clenshaw-Curtis quadrature formula is the formula (2.2) based on these nodes. A better name might have been "Chebyshev" or "Fejér" - indeed, Clenshaw and Curtis call it "the Chebyshev formula" - but the term Clenshaw-Curtis is standard.

Clenshaw and Curtis published their paper in 1960, before the introduction of the Fast Fourier Transform in 1965. Soon afterwards, the connection with the FFT was pointed out by Gentleman [21]. Again we shall not give details but offer a MATLAB code with the same functionality as gauss.m:

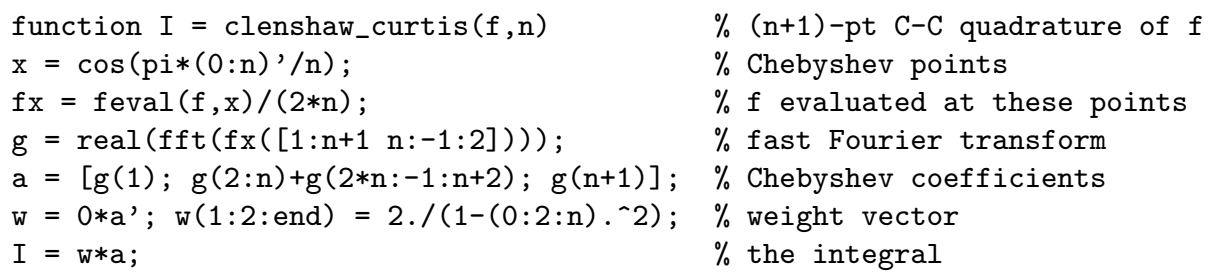

\footnotetext{
${ }^{1}$ Here are Stroud and Secrest in 1966, working on a Control Data 1604: "The formula for $n=512$ took about 4.5 hours to calculate and 0.5 hour to check" [39]. Thus in forty years we have a speedup from hours on a mainframe to seconds on a laptop.

${ }^{2}$ For FFT evaluations of the other two rules, see [44].
} 
To get full accuracy for the cosine example, we now need clenshaw_curtis (@cos,11). Increasing $n$ from 11 to $10^{3}$ or $10^{6}$ gives the same result. The former runs in less than a millisecond on my laptop, and the latter takes 1.6 seconds.

From the point of view of integrating polynomials exactly, Clenshaw-Curtis nodes appear like Newton-Cotes nodes: both are exact merely for degree $n$. Yet it is clear from Figure 2.1 that in a literal sense, at least, they are closer to Gauss nodes. Certainly they have the same asymptotic distribution as $n \rightarrow \infty$, the density $n \pi^{-1}\left(1-x^{2}\right)^{-1 / 2}$ that is well known to be optimal in various senses for polynomial approximation on $[-1,1][41$, chap. 5].

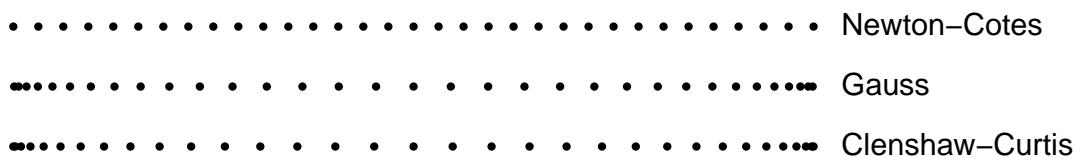

FIG. 2.1. Newton-Cotes, Gauss, and Clenshaw-Curtis quadrature points in $[-1,1]$ for $n=32$. The latter two sets have asymptotically the same clustering near \pm 1 as $n \rightarrow \infty$.

3. Experimental comparison. Figure 3.1 shows the convergence as $n \rightarrow \infty$ of the Gauss and Clenshaw-Curtis formulas for six functions $f(x)$. (This experiment is adapted from Outputs 30b and 30c of [41].) We begin with the monomial $f(x)=x^{20}$, the algebraic analogue of what in the periodic context would be called a band-limited function. Here the factor of 2 is in one sense clearly visible: the Gauss formula is exact for $n \geq 10$, the Clenshaw-Curtis formula for $n \geq 20$. Even so, it is apparent that for smaller values of $n$, the Gauss advantage in efficiency (i.e., size of $n$ needed to achieve a certain accuracy) is less than a factor of 2 . The second function is $e^{x}$, which is entire, i.e., analytic throughout the complex plane. Here again Gauss appears more efficient than Clenshaw-Curtis, but by less than a factor of 2 .

Given $f \in C[-1,1]$ and $n \geq 0$, let $p_{n}^{*}$ be the unique best approximation to $f$ on $[-1,1]$ of degree $\leq n$ with respect to the supremum norm $\|\cdot\|=\|\cdot\|_{\infty}$, and define $E_{n}^{*}=\left\|f-p_{n}^{*}\right\|$. The solid curves in each panel of Figure 3.1 show the errors $E_{n}^{*}$ and $E_{2 n+1}^{*}$. (We computed these numbers via Carathéodory-Fejér approximation [23] using the chebfun system [2], a convenient environment for all kinds of explorations related to this paper.) We shall analyze these curves in the next section, but for now, we mention just the essential point: Clenshaw-Curtis quadrature converges faster than $E_{n}^{*}$.

The next four functions are successively less smooth. The third is $e^{-x^{2}}$, which is also entire, but now with stronger growth $O\left(e^{|x|^{2}}\right)$ as $x \rightarrow \infty$ in the complex plane. Here again we see a difference between Gauss and Clenshaw-Curtis quadrature, but with an efficiency ratio less than 2. The fourth function is $f(x)=\left(1+16 x^{2}\right)^{-1}$, which is analytic in a neighborhood of $[-1,1]$ but not throughout the complex plane, and now we see that the two quadrature formulas are quite close together. (This example is discussed further in §6.) For the last two functions, there is again little difference between Gauss and Clenshaw-Curtis quadrature. We see this both for the $C^{\infty}$ example $e^{-1 / x^{2}}$ and for the non-smooth function $|x|^{3}$.

These and similar experiments suggest the following conclusions: 



FIG. 3.1. Convergence in floating-point arithmetic of Gauss (dots) and Clenshaw-Curtis (circles) quadrature for six functions $f$ on $[-1,1]$, for $n$ ranging from 1 to 30 . The first function is polynomial, the second and third entire, the fourth analytic, the fifth $C^{\infty}$, and the sixth $C^{2}$. The solid curves show the minimax approximation errors $E_{n}^{*}$ (upper) and $E_{2 n+1}^{*}$ (lower).

- As expected, Gauss quadrature matches best degree $2 n+1$ polynomial approximation: $\left|I-I_{n}\right| \approx E_{2 n+1}^{*}$.

- However, Clenshaw-Curtis quadrature does much better than $\left|I-I_{n}\right| \approx E_{n}^{*}$. In fact, for functions that are not analytic in a large neighborhood of $[-1,1]$, it too comes close to $\left|I-I_{n}\right| \approx E_{2 n+1}^{*}$.

- Thus Gauss quadrature significantly outperforms Clenshaw-Curtis quadrature only for functions analytic in a large neighborhood of $[-1,1]$.

- For such functions, the convergence of both methods is very fast. Thus Clenshaw-Curtis essentially never requires many more function evaluations than Gauss to converge to a prescribed accuracy.

We are not the first to observe these surprises. Clenshaw and Curtis themselves recorded the same effect:

It may be of interest to note that a program based on the proposed method has been prepared for the Deuce at the National Physical Labo- 


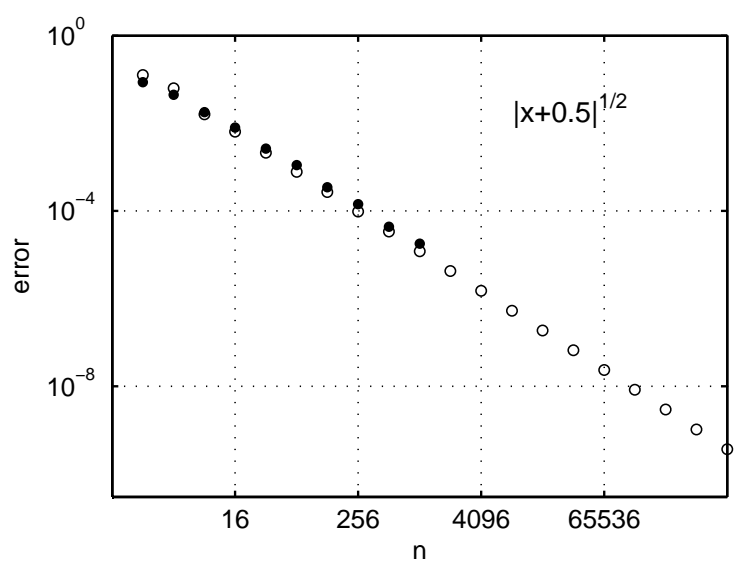

FIG. 3.2. Convergence of Gauss (dots) and Clenshaw-Curtis (circles) quadrature for the example $f(x)=\left|x+\frac{1}{2}\right|^{1 / 2}$ of Clenshaw and Curtis, shown on log-log axes. The Gauss formula is applied only up to $n=2^{10}$, since larger values take too much time and memory.

ratory. The upper limit $M$ of the number of terms is 64 , and the program evaluated the integral $\int_{-1}^{+1}\left|x+\frac{1}{2}\right|^{\frac{1}{2}} d x$ with an error of 0.00078 . This may be compared with the error of 0.00317 committed by the 32-point Gauss formula, and of 0.00036 by the 64 -point Gauss formula. We see that the Chebyshev formula, which is much more convenient than the Gauss, may sometimes nevertheless be of comparable accuracy.

Figure 3.2 examines this function considered by Clenshaw and Curtis for a collection of values of $n$. There is no advantage whatever for Gauss quadrature. (The reason the plot does not match the numbers they report is that it shows data for $n=64$ whereas their numbers correspond to $n=63$.)

A 1968 paper of O'Hara and Smith makes an unqualified statement [35]:

... the Clenshaw-Curtis method gives results nearly as accurate as Gaussian quadratures for the same number of abscissae.

4. Quadrature, polynomial approximation, and Chebyshev expansions. How can we explain these observations? In the next section we shall see that some of the answers can be found in the work in the 1960s of O'Hara and Smith, quoted above, and Elliott [12]. To prepare the way, we begin with a discussion of some connections between quadrature formulas, polynomial approximations, and Chebyshev expansions. Though the flavor of these developments is standard, the precise estimates we give are sharper than those we have found in the literature: this applies to (4.6), (4.10), (4.12), (4.13), and (4.14), as well as the Jackson theorem of footnote 4. We consider Chebyshev expansions for two reasons: first, because they provide nearbest polynomial approximations and thus near-sharp bounds on Gauss quadrature errors; second, because they have a special connection with Clenshaw-Curtis quadrature that will lead to an explanation of its surprising accuracy.

Given a continuous function $f$ on $[-1,1]$, let the degree $\leq n$ polynomial best approximation $p_{n}^{*}$ and error $E_{n}^{*}$ be defined as in the last section. The rationale for including the solid curves in Figure 3.1 is the following result due to Bernstein [3].

THEOREM 4.1. If the weights $w_{k}$ in $(2.2)$ are nonnegative, as is true for both 
Gauss and Clenshaw-Curtis quadrature, then for any $f \in C[-1,1]$ and $n \geq 0$,

$$
\left|I-I_{n}\right| \leq 4 E_{n}^{*}
$$

and $I_{n} \rightarrow I$ as $n \rightarrow \infty$. In the special case of Gauss quadrature, (4.1) can be improved to

$$
\left|I-I_{n}\right| \leq 4 E_{2 n+1}^{*}
$$

Proof. We take advantage of the fact that since (2.2) integrates polynomials of degree $\leq n$ exactly, $I$ and $I_{n}$ are the same for $f-p_{n}^{*}$ as for $f$ itself. From (2.2), using the fact that the interval $[-1,1]$ has length 2 , we thus have

$$
\begin{aligned}
\left|I(f)-I_{n}(f)\right| & =\left|I\left(f-p_{n}^{*}\right)-I_{n}\left(f-p_{n}^{*}\right)\right| \\
& \leq\left|I\left(f-p_{n}^{*}\right)\right|+\left|I_{n}\left(f-p_{n}^{*}\right)\right| \leq 2 E_{n}^{*}+\sum_{k=0}^{n}\left|w_{k}\right| E_{n}^{*} .
\end{aligned}
$$

Since the quadrature formula is interpolatory, $\sum w_{k}=2$, and if the weights are nonnegative, this implies $\sum\left|w_{k}\right|=2$, from which (4.1) follows, and similarly (4.2) in the case of Gauss quadrature since in that case $p_{n}^{*}$ can be replaced by $p_{2 n+1}^{*}$. The positivity of the weights was proved for Gauss quadrature by Stieltjes in 1884, and for Clenshaw-Curtis quadrature by Imhof in 1963 [24]. The convergence of $I_{n}$ to $I$ is a consequence of (4.1) together with the Weierstrass approximation theorem.

Theorem 4.1 tells us that if the best approximants to $f$ converge rapidly as $n \rightarrow \infty$, then $I_{n}$ will converge rapidly to $I$. The next step is to combine this theorem with results of approximation theory to the effect that if $f$ is smooth, its best approximants converge rapidly. We shall consider some results of this kind derived from the Chebyshev series for a function $f \in C[-1,1]$. The logic of the development can be summarized like this:

$$
\begin{gathered}
\text { smooth function } f \\
\Downarrow \\
\text { rapidly decreasing Chebyshev coefficients } \\
\Downarrow \\
\text { rapidly converging Chebyshev partial sums } \\
\Downarrow \\
\text { rapidly converging best approximations } \\
\Downarrow \\
\text { rapidly converging quadrature }
\end{gathered}
$$

We shall also see that sometimes the sharpest results can be obtained by bypassing steps three and four and going directly from the Chebyshev coefficients to the quadrature formulas.

Let $T_{j}$ denote the Chebyshev polynomial of degree $j, T_{j}(x)=\cos \left(j \cos ^{-1} x\right)$, which equioscillates between $j+1$ extrema \pm 1 on $[-1,1]$. The Chebyshev series for $f \in C[-1,1]$ is defined by $[38$, p. 165$]$

$$
f(x)=\sum_{j=0}^{\infty} a_{j} T_{j}(x), \quad a_{j}=\frac{2}{\pi} \int_{-1}^{1} \frac{f(x) T_{j}(x)}{\sqrt{1-x^{2}}} d x
$$


where the prime indicates that the term with $j=0$ is multiplied by $1 / 2$. (These formulas are nothing more than the transplantation to $x=\cos \theta$ of the Fourier series for the $2 \pi$-periodic even function $f(\cos \theta)$.) The equals sign in the first formula is justified, for it is known that the series converges pointwise for any $f \in C[-1,1][31$, p. 119], and if $f$ is Hölder continuous with any Hölder exponent $>0$, the convergence is uniform and absolute [38, p. 168].

Our first step is to show that if $f$ is smooth, its Chebyshev coefficients decrease rapidly. We consider two smoothness conditions: a $k$ th derivative satisfying a condition related to bounded variation, or analyticity in a neighborhood of $[-1,1]$. Regarding the former, let $\|\cdot\|_{T}$ be the Chebyshev-weighted 1-norm defined by

$$
\|u\|_{T}=\left\|\frac{u^{\prime}(x)}{\sqrt{1-x^{2}}}\right\|_{1} .
$$

This norm is defined via a Stieltjes integral for any $u$ of bounded variation (see, e.g., [47, p. 220]), though $\|u\|_{T}$ may be infinite depending on the behavior near $x= \pm 1$. The condition of interest for our theorems is that $\left\|f^{(k)}\right\|_{T}$ should be finite, where $f^{(k)}$ is the $k$ th derivative of $f .^{3}$

THEOREM 4.2. If $f, f^{\prime}, \ldots, f^{(k-1)}$ are absolutely continuous on $[-1,1]$ and $\left\|f^{(k)}\right\|_{T}=$ $V<\infty$ for some $k \geq 0$, then for each $n \geq k+1$,

$$
\left|a_{n}\right| \leq \frac{2 V}{\pi n(n-1) \cdots(n-k)} .
$$

If $f$ is analytic with $|f(z)| \leq M$ in the region bounded by the ellipse with foci \pm 1 and major and minor semiaxis lengths summing to $\rho>1$, then for each $n \geq 0$,

$$
\left|a_{n}\right| \leq \frac{2 M}{\rho^{n}} .
$$

Proof. For (4.7), see [38, p. 175]. As for (4.6), I do not know where this result can be found in print; I am grateful to József Szabados and Endre Süli for suggesting the following argument. We proceed by transplanting to the $\theta=\cos ^{-1} x$ variable and integrating by parts $k+1$ times; the assumptions ensure that the first $k$ derivatives exist in the ordinary sense and that $f^{(k)}$ can in turn be written as a Stieltjes integral. Since $d x=-\sin \theta d \theta$ and $\sqrt{1-x^{2}}=\sin \theta$, the first integration by parts can be written as

$$
\begin{aligned}
a_{n} & =\frac{2}{\pi} \int_{-1}^{1} \frac{f(x) T_{n}(x)}{\sqrt{1-x^{2}}} d x \\
& =\frac{2}{\pi} \int_{0}^{\pi} f(\cos \theta) \cos (n \theta) d \theta \\
& =\frac{2}{\pi n} \int_{0}^{\pi} f^{\prime}(\cos \theta) \sin (n \theta) \sin \theta d \theta ;
\end{aligned}
$$

the boundary terms vanish because of the sine. The trigonometric identity $\sin \theta_{1} \sin \theta_{2}=$ $\frac{1}{2} \cos \left(\theta_{1}-\theta_{2}\right)-\frac{1}{2} \cos \left(\theta_{1}+\theta_{2}\right)$ converts this to

$$
a_{n}=\frac{2}{\pi n} \int_{0}^{\pi} f^{\prime}(\cos \theta)\left[\frac{\cos ((n-1) \theta)}{2}-\frac{\cos ((n+1) \theta)}{2}\right] d \theta
$$

\footnotetext{
${ }^{3}$ Our attention to this condition derives from a moral principle: the exponent should come out right for the function $f(x)=|x|$. For this function we can take $k=1$ in Theorem 4.2, giving $a_{n}=O\left(n^{-2}\right)$.
} 
which implies

$$
\begin{aligned}
\left|a_{n}\right| & \leq \frac{2}{\pi n}\left\|f^{\prime}(\cos \theta)\right\|_{1}\left\|\frac{\cos ((n-1) \theta)}{2}-\frac{\cos ((n+1) \theta)}{2}\right\|_{\infty} \\
& =\frac{2}{\pi n}\left\|f^{\prime}(x)\right\|_{T}\left\|\frac{\cos ((n-1) \theta)}{2}-\frac{\cos ((n+1) \theta)}{2}\right\|_{\infty}
\end{aligned}
$$

since $d \theta=d x / \sqrt{1-x^{2}}$. The $L^{\infty}$ norm is bounded by 1 , and thus we have established $\left|a_{n}\right| \leq 2 V^{(0)} / \pi n$ with $V^{(0)}=\|f\|_{T}$. Further integrations by parts bring in higher derivatives of $f$ and corresponding higher variations up to $V^{(k)}=V=\left\|f^{(k)}\right\|_{T}$. More and more cosine terms appear, but the coefficients are such that their sum always has $L^{\infty}$ norm at most 1. Just as the first integration by parts introduced a factor $n$ in the denominator, the second leads to factors $n-1$ and $n+1$, the third to factors $n-2, n$, and $n+2$, and so on. To keep the formulas simple we do not keep track of all these different denominators but weaken the inequality slightly by replacing them all with $n-1$ at the second differentiation, $n-2$ at the third, and so on up to $n-k$ at the $(k+1)$ st differentiation. The result is $(4.6)$.

Our next step is to consider polynomial approximations obtained as partial sums of the Chebyshev series. For any $n \geq 0$, define

$$
f_{n}(x)=\sum_{j=0}^{n} a_{j} T_{j}(x)
$$

and

$$
E_{n}^{T}=\left\|f-f_{n}\right\| \leq \sum_{j=n+1}^{\infty}\left|a_{j}\right| .
$$

(The polynomial $f_{n}$ can be interpreted as the least-squares projection of $f$ onto the set of polynomials of degree $\leq n$ with respect to the weight $\left(1-x^{2}\right)^{-1 / 2}$. It can also be obtained from $f$ by convolution in the $\theta$ variable with a certain $n$-dependent kernel [38, p. 166].) The following theorem is a corollary of Theorem 4.2 and equation (4.9).

Theorem 4.3. If $f, f^{\prime}, \ldots, f^{(k-1)}$ are absolutely continuous on $[-1,1]$ and $\left\|f^{(k)}\right\|_{T}=$ $V<\infty$ for some $k \geq 1$, then for each $n \geq k+1$,

$$
E_{n}^{T} \leq \frac{2 V}{\pi k(n-k)^{k}} .
$$

If $f$ is analytic with $|f(z)| \leq M$ in the region bounded by the ellipse with foci \pm 1 and major and minor semiaxis lengths summing to $\rho>1$, then for each $n \geq 0$,

$$
E_{n}^{T} \leq \frac{2 M}{(\rho-1) \rho^{n}}
$$

Proof. Equation (4.11) follows readily from (4.7) and (4.9). (This bound is erroneously reported without the factor of 2 on p. 133 of [31].) Similarly, (4.10) follows from (4.6) and (4.9) if we use the inequality

$$
\sum_{j=n+1}^{\infty} \frac{1}{j(j-1) \cdots(j-k)} \leq \sum_{j=n+1}^{\infty} \frac{1}{(j-k)^{k+1}} \leq \int_{n}^{\infty} \frac{d x}{(x-k)^{k+1}}=\frac{1}{k(n-k)^{k}} .
$$


Now how does $E_{n}^{T}$, the error of the truncated Chebyshev series, compare with the best approximation error $E_{n}^{*}$ ? In one direction the answer is obvious, since the optimality of $E_{n}^{*}$ implies $E_{n}^{*} \leq E_{n}^{T} .{ }^{4}$ In the other direction it is well known that the gap is never bigger than $O(\log n)$. The following theorem states this result together with another lower bound on $E_{n}^{*}$.

TheOREM 4.4. For any $f \in C[-1,1]$ and $n \geq 0$,

$$
\frac{\pi}{4}\left|a_{n+1}\right| \leq E_{n}^{*} \leq E_{n}^{T} \leq\left(2+\frac{4}{\pi^{2}} \log (2 n+1)\right) E_{n}^{*} .
$$

In particular, $E_{n}^{T}<4.2 E_{n}^{*}$ for $n \leq 100$ and $E_{n}^{T}<7.9 E_{n}^{*}$ for $n \leq 10^{6}$.

Proof. The first inequality is due to Bernstein; see [6, p. 131] or [38, p. 171]. The last inequality follows from the fact that the Lebesgue constant for Chebyshev projection, which is the same as the Lebesgue constant for Fourier projection defined originally by Lebesgue (1909), is bounded by $1+\left(4 / \pi^{2}\right) \log (2 n+1)$ [18]. The $\sim\left(4 / \pi^{2}\right) \log n$ dependence was known already to Fejér (1910) and Bernstein (1912).

By combining Theorems 4.1 and 4.3 , we get bounds on $I-I_{n}$, which Theorem 4.4 indicates are likely to be reasonably sharp. Here are bounds for Gauss quadrature.

TheOREM 4.5. Let Gauss quadrature be applied to a function $f \in C[-1,1]$. If $f, f^{\prime}, \ldots, f^{(k-1)}$ are absolutely continuous on $[-1,1]$ and $\left\|f^{(k)}\right\|_{T}=V<\infty$ for some $k \geq 1$, then for each $n \geq k / 2$,

$$
\left|I-I_{n}\right| \leq \frac{64 V}{15 \pi k(2 n+1-k)^{k}} .
$$

If $f$ is analytic with $|f(z)| \leq M$ in the region bounded by the ellipse with foci \pm 1 and major and minor semiaxis lengths summing to $\rho>1$, then for each $n \geq 0$,

$$
\left|I-I_{n}\right| \leq \frac{64 M}{15(\rho-1) \rho^{2 n+1}} .
$$

Partial proof. Suppose the factors $64 / 15$ in these statements are increased to 8 . Then (4.13) follows from (4.2) and (4.10), and (4.14) from (4.2) and (4.11). The completion of the proof by sharpening the constants to $64 / 15$ is discussed in the next section. ${ }^{5} \mathrm{c}$

The bounds of Theorem 4.5 are valid for Clenshaw-Curtis quadrature, except with $2 n+1$ replaced by $n$. However, we do not write these inequalities down, as we have seen that they are highly pessimistic and our aim is to explain that effect. Ideally, we would like to establish bounds for Clenshaw-Curtis quadrature that are not much different from those of Theorem 4.5 -i.e., with " $2 n$ ", not " $n$ ". The next section will present such an extension to Clenshaw-Curtis quadrature of (4.13), and Section 6 will point the way toward a possible extension of (4.14).

\footnotetext{
${ }^{4}$ Thus (4.10) provides a proof of a variant of one of the Jackson theorems of approximation theory [6, p. 147]: $E_{n}^{*} \leq 2 V / \pi k(n-k)^{k}$.

${ }^{5}$ Bounds based on the assumption of analyticity in an ellipse were first derived by Bernstein [3]; for a discussion, see [20, p. 114]. According to Gautschi, the sharpest result as of 1981 was one derived by von Sydow, in which $64 / 15$ is replaced in $(4.14)$ by $8 \rho /(\rho+1)$ [43]. For $\rho>8 / 7$, the bound (4.14) is better.
} 
5. Explanation 1: Chebyshev expansions and aliasing. Let us immediately state our conclusion: the first half of Theorem 4.5 holds for Clenshaw-Curtis as well as Gauss quadrature. The sole change needed is to replace the condition $n \geq k / 2$ by the condition of sufficiently large $n$. Thus Clenshaw-Curtis has essentially the same algebraic rate of convergence as Gauss quadrature for several-times-differentiable functions.

THEOREM 5.1. Let Clenshaw-Curtis quadrature be applied to a function $f \in$ $C[-1,1]$. If $f, f^{\prime}, \ldots, f^{(k-1)}$ are absolutely continuous on $[-1,1]$ and $\left\|f^{(k)}\right\|_{T}=V<$ $\infty$ for some $k \geq 1$, then for all sufficiently large $n$,

$$
\left|I-I_{n}\right| \leq \frac{64 V}{15 \pi k(2 n+1-k)^{k}}
$$

"Sufficiently large $n$ " means $n>n_{k}$ for some $n_{k}$ that depends on $k$ but not $f$ or $V$.

Notice the key implication: the factor $2^{-k}$ in the error bound (4.13) for Gauss quadrature, which one thinks of as coming from its doubled order of accuracy, applies to Clenshaw-Curtis quadrature too. In this section we shall derive this bound. The theorem is new, but it is built on the ideas of Elliott [12] and O'Hara and Smith [35] forty years ago.

The crucial fact is that of aliasing. Elementary algebra shows that on the grid in $[0,2 \pi]$ of $2 n$ equally spaced points $\theta_{j}=\pi j / n, 0 \leq j \leq 2 n-1$, the functions $\cos \left((n+p) \pi \theta_{j}\right)$ and $\cos \left((n-p) \pi \theta_{j}\right)$ are indistinguishable for any integer $p$. We say that the wave numbers $(n+p) \pi$ and $(n-p) \pi$ are aliases of one another on this grid. Transplanting to the variable $x=\cos \theta$ gives the following result.

Theorem 5.2. For any integer $p$ with $0 \leq p \leq n$,

$$
T_{n+p}\left(x_{j}\right)=T_{n-p}\left(x_{j}\right) \quad(0 \leq j \leq n)
$$

on the Chebyshev grid (2.3). Consequently Clenshaw-Curtis quadrature gives the same result for both functions,

$$
I_{n}\left(T_{n+p}\right)=I_{n}\left(T_{n-p}\right)=I\left(T_{n-p}\right)=\left\{\begin{array}{ll}
0 & \text { if } n \pm p \text { is odd } \\
\frac{2}{1-(n-p)^{2}} & \text { if } n \pm p \text { is even }
\end{array},\right.
$$

and the error in integrating $T_{n+p}$ is

$$
I\left(T_{n+p}\right)-I_{n}\left(T_{n+p}\right)=\left\{\begin{array}{ll}
0 & \text { if } n \pm p \text { is odd } \\
\frac{8 p n}{n^{4}-2\left(p^{2}+1\right) n^{2}+\left(p^{2}-1\right)^{2}} & \text { if } n \pm p \text { is even }
\end{array} .\right.
$$

Proof. The equality (5.2) was established by the discussion above it. Since Clenshaw-Curtis quadrature is exact for polynomials of degree $\leq n$, the identity (5.3) follows from the standard formula $\int_{-1}^{1} T_{j}(x) d x=2 /\left(1-j^{2}\right)$ for the integral of a Chebyshev polynomial of even order (which appeared in the penultimate line of clenshaw_curtis in Section 2). Finally, taking the difference of the integrals with $j=n+p$ and $j=n-p$ gives (5.4).

From (5.4) we can see why Clenshaw-Curtis quadrature is unexpectedly accurate. Suppose $n$ is even for simplicity. Then the first few terms in the Chebyshev expansion of $f$ that contribute to the error $I(f)-I_{n}(f)$ are

$$
a_{n+2} T_{n+2}, a_{n+4} T_{n+4}, \ldots
$$


However, the exact integrals of these Chebyshev polynomials are small, of order $O\left(n^{-2}\right)$, and thanks to aliasing, their values as computed by Clenshaw-Curtis quadrature will also be small. From (5.4), we see that if $n$ is large, the errors associated with the terms above are approximately

$$
\frac{16}{n^{3}}\left|a_{n+2}\right|, \frac{32}{n^{3}}\left|a_{n+4}\right|, \ldots
$$

The numbers grow as the subscript increases from $n$ towards $2 n$, but even for a "typical" term $a_{n+p}$, the error will be of order $n^{-2}$. For a numerical illustration, suppose $n=50$. Then the $(n+1)$-point Clenshaw-Curtis formula integrates $T_{52}$ inexactly, but the error is only about 0.0001. Similarly its errors for $T_{60}, T_{70}, T_{80}$, and $T_{90}$ are about $0.0006,0.002,0.006$, and 0.02, respectively. By contrast the Gauss formula with $n=50$ integrates all the polynomials up to $T_{101}$ exactly, but after that its error jumps immediately to order 1 : for $T_{102}$, about -1.6 .

We can now derive (5.1), and in the process, explain how the constant 8 in our partial proof of Theorem 4.5 can be improved to $64 / 15$.

Proof of Theorem 5.1 and completion of proof of Theorem 4.5. From the Chebyshev expansion of $f$ we know that the quadrature error can be written

$$
I(f)-I_{n}(f)=\sum_{j=0}^{\infty} a_{j}\left(I\left(T_{j}\right)-I_{n}\left(T_{j}\right)\right) .
$$

This implies

$$
\left|I(f)-I_{n}(f)\right| \leq S_{1}+S_{2}+S_{3}+S_{4}
$$

where

$$
\begin{aligned}
S_{1} & =\sum_{j=0}^{n}\left|a_{j}\right|\left|I\left(T_{j}\right)-I_{n}\left(T_{j}\right)\right|, \\
S_{2} & =\sum_{j=n+1}^{2 n-\left\lfloor n^{1 / 3}\right\rfloor}\left|a_{j}\right|\left|I\left(T_{j}\right)-I_{n}\left(T_{j}\right)\right|, \\
S_{3} & =\sum_{j=2 n+1-\left\lfloor n^{1 / 3}\right\rfloor}^{2 n+1}\left|a_{j}\right|\left|I\left(T_{j}\right)-I_{n}\left(T_{j}\right)\right|, \\
S_{4} & =\sum_{j=2 n+2}^{\infty}\left|a_{j}\right|\left|I\left(T_{j}\right)-I_{n}\left(T_{j}\right)\right| .
\end{aligned}
$$

The term $S_{1}$ is zero since the quadrature formula is interpolatory. From (5.4) it can be seen that the factors $\left|I\left(T_{j}\right)-I_{n}\left(T_{j}\right)\right|$ that appear in $S_{2}$ are all of order at worst $n^{-2 / 3}$, and by Theorem 4.2 , the coefficients $a_{j}$ are of order $V n^{-k-1}$. Thus $S_{2}$ consists of $O(n)$ terms of size $O\left(V n^{-k-5 / 3}\right)$, for a total magnitude $O\left(V n^{-k-2 / 3}\right)$. The term $S_{3}$ consists of $O\left(n^{1 / 3}\right)$ terms of size $O\left(V n^{-k-1}\right)$, for a total magnitude again $O\left(V n^{-k-2 / 3}\right)$. Finally, consider $S_{4}$. The partial proof of Theorem 4.5 given in the last section implies a bound of $8 V / \pi k(2 n+1-k)^{k}$, which is what we would get again now if we estimated $S_{4}$ by applying the bound (4.6) for $a_{j}$ together with the inequality $\left|I\left(T_{j}\right)-I_{n}\left(T_{j}\right)\right| \leq 4$. However, since $j \geq 4$, from (5.2) or (5.3) it can be seen that we actually have $\left|I\left(T_{j}\right)-I_{n}\left(T_{j}\right)\right| \leq 2+2 / 15=32 / 15$. It is this observation that sharpens 
the constant in Theorem 4.5. For Theorem 5.1, we have the freedom to increase $n$ a little further. In particular, the assumption $n \geq 2$ is enough to imply $j \geq 6$, further shrinking the constant to $2+2 / 35=72 / 35$. Combining this with (4.6) and putting the pieces together, we find

$$
S_{1}+S_{2}+S_{3}+S_{4} \leq O\left(V n^{-k-2 / 3}\right)+\frac{144 V / 35}{\pi k(2 n+1-k)^{k}}<\frac{64 V / 15}{\pi k(2 n+1-k)^{k}}
$$

for large enough $n$. $\square$

6. Explanation 2: rational approximation of $\log ((z+1) /(z-1))$. So far, I have not mentioned why this article came to be written. The answer is about to appear: Figures 6.1 and 6.2. Some time ago I undertook to investigate analogues for $[-1,1]$ of observations put forward in [42] about quadrature and rational approximation on $(-\infty, 0]$. As soon as these figures appeared, I knew there was a story to be told about Clenshaw-Curtis quadrature.

To set the stage, we turn to an old idea for estimating the accuracy of quadrature formulas applied to analytic functions: Cauchy integrals in the complex plane. As always, we are interested in the relationship of the integral $I$ of (2.1) to its discrete approximation $I_{n}$ of $(2.2)$ for a function $f \in C[-1,1]$. Suppose that $f$ is analytic in a neighborhood of $[-1,1]$, and let $\Gamma$ be a contour in the region of analyticity that winds once around $[-1,1]$ in the counterclockwise direction. Then using Cauchy's integral formula we can write

$$
I=\int_{-1}^{1} f(x) d x=\int_{-1}^{1} \frac{1}{2 \pi i} \int_{\Gamma} \frac{f(z)}{z-x} d z d x=\frac{1}{2 \pi i} \int_{\Gamma} f(z) \phi(z) d z
$$

where $\phi(z)$ is given by

$$
\phi(z)=\int_{-1}^{1} \frac{d x}{z-x}=\log \frac{z+1}{z-1} .
$$

To be precise, $\phi$ is defined as the analytic function in $\mathbb{C} \cup\{\infty\} \backslash[-1,1]$ given by the branch of $\log ((z+1) /(z-1))$ that is positive for $z \in(1, \infty)$. At the same time, using complex residue calculus, we can also express $I_{n}$ as a contour integral over $\Gamma$ :

$$
I_{n}=\sum_{k=0}^{n} w_{k} f\left(x_{k}\right)=\frac{1}{2 \pi i} \int_{\Gamma} f(z) r_{n}(z) d z,
$$

where $r_{n}$ is defined by

$$
r_{n}(z)=\sum_{k=0}^{n} \frac{w_{k}}{z-x_{k}}
$$

Combining (6.1) and (6.3) now gives a beautiful result.

THEOREM 6.1. Let $f$ be analytic in a neighborhood of $[-1,1]$ and let $\Gamma$ be a contour contained in the region of analyticity of $f$ that encloses $[-1,1]$ once in the counterclockwise direction. Let $\phi$ be defined by (6.2), and for any $n \geq 0$, let $I_{n}$ be the result of an arbitrary quadrature formula (2.2) with nodes in $[-1,1]$. Then the error is given by

$$
I-I_{n}=\frac{1}{2 \pi i} \int_{\Gamma} f(z)\left(\phi(z)-r_{n}(z)\right) d z
$$


where $r_{n}$ is defined by (6.4), and hence

$$
\left|I-I_{n}\right| \leq \frac{1}{2 \pi}|\Gamma|\|f\|_{\Gamma}\left\|\phi-r_{n}\right\|_{\Gamma},
$$

where $|\Gamma|$ is the arc length of $\Gamma$ and $\|\cdot\|_{\Gamma}$ is the supremum norm over $\Gamma$.

This theorem shows that quadrature over $[-1,1]$ is associated with a problem in rational approximation: how closely can $\phi(z)=\log ((z+1) /(z-1))$ be approximated in a region of the complex plane near $[-1,1]$ by a rational function of type $(n, n+1)$, i.e., with numerator of degree $\leq n$ and denominator of degree $\leq n+1$ ? Note that $\phi(\infty)=0$, so it makes sense that the degree of the denominator exceeds that of the numerator. Any sum (6.4) with nonzero weights $w_{k}$ and distinct nodes $x_{k}$ defines a rational function of type $(n, n+1)$, and conversely, if a rational function of type $(n, n+1)$ is expanded in partial fractions, it will have the form (6.4) provided it has $n+1$ distinct poles.

The idea of relating quadrature formulas to rational functions goes back to Gauss himself $[19,20]$. In fact, Gauss's original derivation is closer to this way of thinking than many of the subsequent developments framed in terms of orthogonal polynomials by Jacobi, Christoffel, Stieltjes and others. Gauss quadrature formulas have a wonderfully simple characterization in terms of rational approximants:

THEOREM 6.2. For any $n \geq 0$, the rational function $r_{n}$ of (6.4) associated with the $(n+1)$-point Gauss quadrature formula is the type $(n, n+1)$ Padé approximant to $\phi(z)$ at $z=\infty$. The function $\phi-r_{n}$ has a zero of order exactly $2 n+3$ at $z=\infty$.

Proof. Gauss derived this result by continued fractions [19]; see [20, §1.2].

A Padé approximant, as always, is defined as the unique rational function of the specified type that matches the Taylor expansion of the given function at the given point, here $\infty$, to as high an order as possible. This terminology was not available in Gauss's day, but Theorem 6.2 is his nonetheless.

What about Clenshaw-Curtis quadrature?

We begin with an experimental comparison. Figure 6.1 shows errors $\left|\left(\phi-r_{n}\right)(z)\right|$ as contour plots in the complex plane for Newton-Cotes, Gauss, and Clenshaw-Curtis formulas with $n=8$ and 16 . In each case the innermost contour corresponds to $\left|\phi(z)-r_{n}(z)\right|=1$, and moving outward, the levels are $10^{-1}, 10^{-2}, \ldots, 10^{-13}$. As $z \rightarrow \infty$, all the approximations become very good. Figures like these have been drawn previously by Takahasi and Mori [40], for Gauss quadrature but not Clenshaw-Curtis, as part of an important series of papers on quadrature formulas and their enhancement by changes of variables like the double exponential transformation. Among other contributions, Takahasi and Mori state Theorem 6.1. The only other publication that I know of where figures like these appear is [42], for quadrature over $(-\infty, 0]$.

What can we infer from such images? Suppose $f$ is analytic in a big region of the complex plane and does not grow too fast as $|z|$ increases. Then (6.6) implies that we may get a small bound on $\left|I-I_{n}\right|$ by integrating over one of the contour levels far from $[-1,1]$. In the extreme case, suppose $f$ is a polynomial of degree $k$. Then Theorem 6.2 implies that for Gauss quadrature, $\left(\phi-r_{n}\right) f$ has a zero of order $2 n+3-k$ at $\infty$. If this number is at least 2 , i.e., $k \leq 2 n+1$, then by taking circular contours $\Gamma$ of radius $R \rightarrow \infty$ we get arbitrarily small bounds on $I-I_{n}$, confirming the exactness of Gauss quadrature for polynomials of degree $\leq 2 n+1$. By reversing the argument we can see that for any interpolatory formula, including Clenshaw-Curtis or Newton-Cotes, $\phi-r_{n}$ must have a zero of order at least $n+2$ at $\infty$. If it did not, taking a large circular contour $\Gamma$ in (6.5) would imply a nonzero error $I-I_{n}$ for some monomial $f(x)=x^{k}$ with $k \leq n$. 

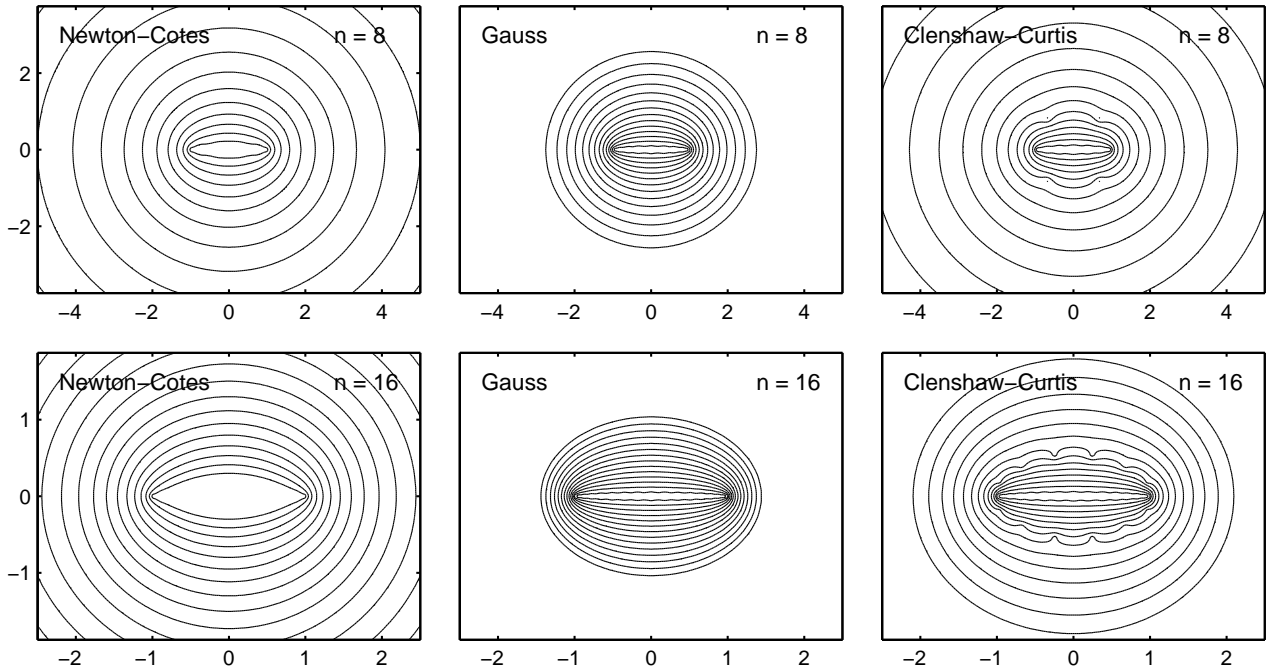

FIG. 6.1. Contour plots of the error $\left|\phi(z)-r_{n}(z)\right|$ for the rational approximations associated with Newton-Cotes, Gauss, and Clenshaw-Curtis quadrature formulas with $n=8$ and 16 . The contours, from inner to outer, correspond to $10^{0}, 10^{-1}, \ldots, 10^{-13}$. The Gauss and Clenshaw-Curtis errors are virtually identical near $[-1,1]$, explaining their similar accuracy for functions that are not analytic in a large region of the plane.

At the other extreme, suppose $f$ is analytic in only a small neighborhood of $[-1,1]$. Then Figure 6.1 suggests that Newton-Cotes quadrature will not converge at all, which is indeed the case. But the remarkable thing is what it suggests about Clenshaw-Curtis quadrature. The figure reveals that near $[-1,1]$, the ClenshawCurtis rational function is essentially indistinguishable from the Gauss rational function as an approximation to $\phi(z)$. Thus we can expect that for a function $f$ of this kind, the two quadrature formulas should have about the same accuracy - exactly what was observed in the last three sections.

Figure 6.2 shows a closeup for the larger value $n=64$. On the left we see contours for Gauss quadrature, and on the right, Clenshaw-Curtis. Now the pattern is even more striking. At $z=\infty, r_{n}$ interpolates $\phi$ to order 131 for Gauss quadrature and only order 67 for Clenshaw-Curtis (since $n$ is even, we get one more than the minimal order $n+2)$, and that is why the outer curves are twice as far apart on the right as on the left. The "missing" 64 interpolation points for Clenshaw-Curtis quadrature, however, lie along a football-shaped curve at a certain distance from $[-1,1]$ (actually there are 62 of them, not 64 ). Inside that curve, the two sides of the figure are almost indistinguishable.

The following is a first step towards explaining this effect.

THEOREM 6.3. Let $r_{n}$ be the rational function (6.4) associated with any interpolatory quadrature formula (2.2) with positive weights. Then the function $\phi(z)-r_{n}(z)$ has at least $n+2$ zeros at $z=\infty$ and exactly $2 n+3$ zeros in $\mathbb{C} \cup\{\infty\} \backslash[-1,1]$ if the points \pm 1 are not quadrature nodes, or $2 n+1$ zeros if these points are nodes.

Proof. A proof of the assertion about $z=\infty$ was sketched above. The result about total count of zeros in $\mathbb{C} \cup\{\infty\} \backslash[-1,1]$ can be established by applying the argument principle to $\phi(z)-r(z)$ over a contour $\Gamma$ that encloses $[-1,1]$ sufficiently tightly. Each quadrature node in $(-1,1)$ contributes an decrease in argument by $2 \pi$ as $\Gamma$ passes nearby in the upper half-plane, then another decrease by $2 \pi$ as it passes 


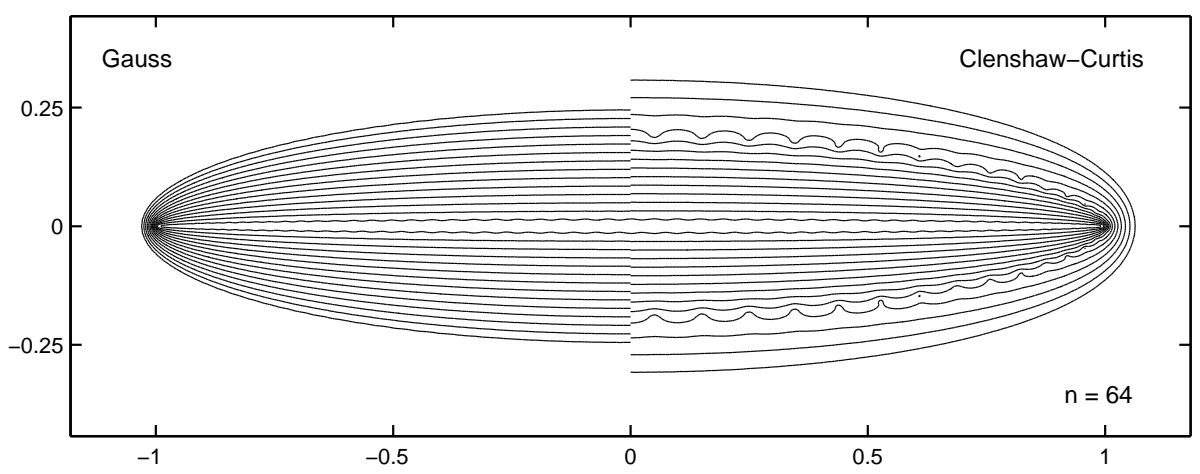

FIG. 6.2. Contour plot of the error $\left|\phi(z)-r_{n}(z)\right|$ as in Figure 6.1, but for $n=64$ and with Gauss on the left half of the image, Clenshaw-Curtis on the right. The scallops in the curves on the right reveal the presence of $n-2$ finite zeros of $\phi(z)-r(z)$ lying along a football-shaped curve surrounding $[-1,1]$.

nearby in the lower half-plane, and the final contributions come from $\phi(z)$, whose argument decreases by $\pi$ near $x=1$ and again by $\pi$ near $x=-1$. We omit the details. $\square$

Thus we see that all interpolatory quadrature formulas with positive weights correspond to rational interpolants to $\phi(z)$ of order approximately $2 n$. What distinguishes Gauss quadrature is that all the interpolation takes place at the single point $\infty$. The rational functions associated with other quadrature formulas can be interpreted as examples of what approximation theorists call multipoint Padé approximants.

Figure 6.3 explores these interpolation points further, showing the $n-2$ zeros of $\phi-r_{n}$ (computed numerically) for four values of $n$. (When $n$ is odd the number of finite zeros increases to $n-1$ and the number of zeros at $z=\infty$ shrinks from $n+3$ to $n+2$.) Two of the interpolation points are real, lying at approximately $\pm\left(1+1.8 n^{-2}\right)$, and the remainder lie in complex conjugate pairs along a curve whose height shrinks approximately at the rate $O\left(n^{-1}\right)$. It is an open problem to analyze the details of this behavior. In particular it would appear that a theorem along the following lines is likely to be valid, which would make an interesting companion to Theorem 5.1: the bound (4.14) applies to Clenshaw-Curtis as well as Guass quadrature so long as $n \leq n_{\rho}$, where $n_{\rho}$ is a number that approaches $\infty$ as $\rho$ shrinks to 1 .

We shall close with an illustration of the implications of Figure 6.3. Consider again the fourth function of Figure 3.1, $f(x)=1 /\left(1+16 x^{2}\right)$. This function is analytic on $[-1,1]$ but has poles at $\pm i / 4$. In Figure 6.3 we see that the curve of interpolation points crosses $\pm i / 4$ somewhere between $n=32$ and $n=64$. This suggests that for values of $n$ larger than about 50, the rate of convergence of the Clenshaw-Curtis formula will cut in half. To test this prediction, Figure 6.4 extends the earlier computation to $n=120$ instead of $n=30$. As expected, there is a kink in the convergence curve at $n \approx 50$. Weideman has given an exact analysis of this example [45].

7. Conclusion. Gauss quadrature, one of the jewels of numerical analysis, is a beautiful and powerful idea. Yet the Clenshaw-Curtis formula has essentially the same performance and can be implemented effortlessly by the FFT. Figure 6.2 offers a memorable explanation of the closeness of these two quadrature formulas. The chebfun system in object-oriented MATLAB takes advantage of the power and speed of Clenshaw-Curtis quadrature to integrate functions defined by as many as millions 

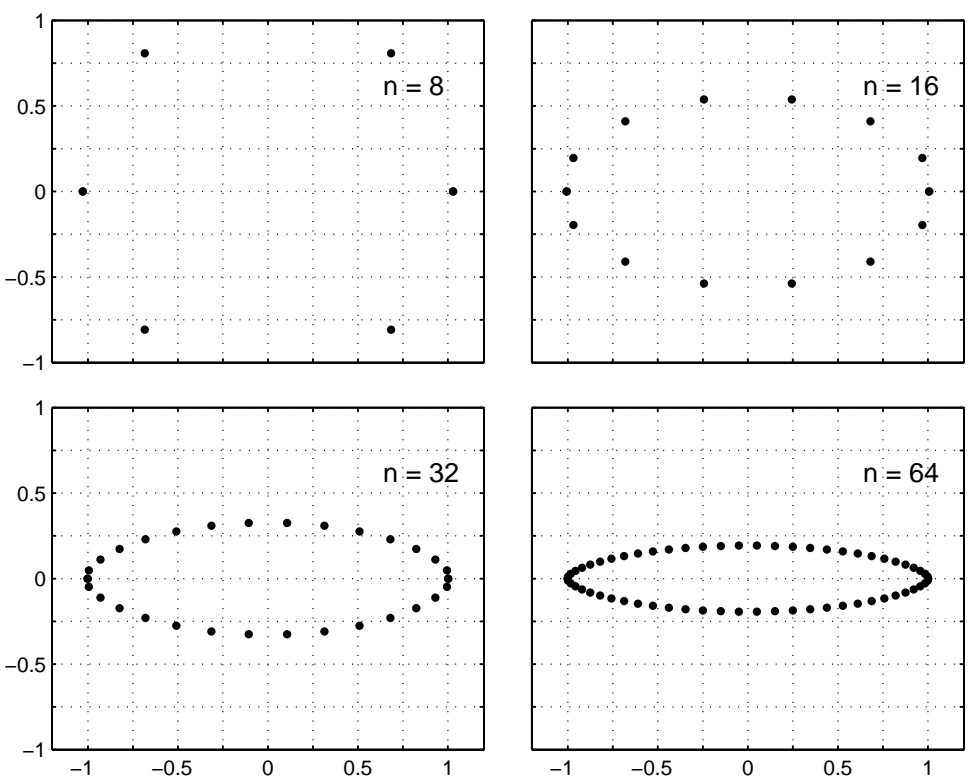

FIG. 6.3. Points in the complex plane at which $\phi(z)-r_{n}(z)=0$ for Clenshaw-Curtis quadrature with $n=8,16,32,64$. In addition there are $n+3$ interpolation points at $z=\infty$.

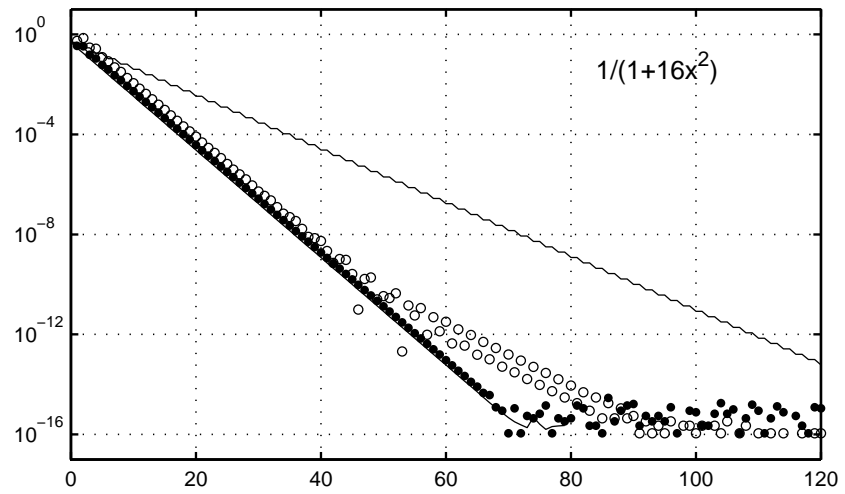

FIG. 6.4. Repetition of the fourth panel of Figure 3.1, but now to the higher value $n=120$. Near $n=50$, the rate of convergence cuts abruptly in half as the curve of interpolation points of Figure 6.3 crosses the poles of $f$ at $\pm i / 4$.

of data points [2].

The observations of this paper may have implications for spectral methods for the numerical solution of ordinary and partial differential equations [4], where the debate between collocation $(\approx$ Clenshaw-Curtis) and Galerkin $(\approx$ Gauss $)$ methods is perennial.

Along the way to our Theorem 5.1 on Clenshaw-Curtis quadrature, we have derived some possibly new results from the consideration of Chebyshev expansions of smooth functions: on decay of Chebyshev coefficients (see eq. (4.6) and footnote 3), on near-optimality of truncated Chebyshev series (eqs. (4.10) and (4.12)), on Jacksontype bounds for polynomial best approximations (footnote 4), and on accuracy of 
Gauss quadrature (eqs. (4.13) and (4.14) and footnote 5).

We hope that others may now take up the challenge of explaining Figures 6.1-6.3 rigorously and establishing corresponding convergence theorems for Clenshaw-Curtis quadrature, perhaps by making use of the potential theoretic notion of "balayage".

Acknowledgments. I have benefitted from the advice of Arno Kuijlaars, Alphonse Magnus, Endre Süli, József Szabados, and André Weideman.

\section{REFERENCES}

[1] W. Barrett, Convergence properties of Gaussian quadrature formulae, Comput. J. 3 (1960/61), 272-277.

[2] Z. Battles and L. N. Trefethen, An extension of MATLAB to continuous functions and operators, SIAM J. Sci. Comp. 25 (2004), 1743-1770.

[3] S. Bernstein, Quelques remarques sur l'interpolation, Math. Ann. 79 (1919), 1-12.

[4] C. Canuto, M. Y. Hussaini, A. Quarteroni and T. A. Zang, Spectral Methods: Fundamentals in Single Domains, Springer, 2006.

[5] M. M. Chawla, Error estimates for the Clenshaw-Curtis quadrature, Math. Comp. 22 (1968), 651-656.

[6] E. W. Cheney, Introduction to Approximation Theory, McGraw-Hill, 1966.

[7] C. W. Clenshaw and A. R. Curtis, A method for numerical integration on an automatic computer, Numer. Math. 2 (1960), 197-205.

[8] P. J. Davies, Errors of numerical approximation for analytic functions, in J. Todd, ed., A Survey of Numerical Analysis, McGraw-Hill, New York, 1962, pp. 468-484.

[9] P. J. Davis and P. Rabinowitz, On the estimation of quadrature errors for analytic functions, Math. Comp. 8 (1954), 193-203.

[10] P. J. Davis and P. Rabinowitz, Methods of Numerical Integration, Academic Press, New York, 1975.

[11] V. A. Dixon. Numerical quadrature; a survey of the available algorithms, in D. J. Evans, ed., Software for Numerical Mathematics, Academic Press, New York, 1974.

[12] D. Elliott, Truncation errors in two Chebyshev series approximations, Math. Comp. 19 (1965), $234-248$.

[13] H. Engels, Numerical Quadrature and Cubature, Academic Press, London, 1980.

[14] G. Evans, Practical Numerical Integration, Wiley, Chichester, 1993.

[15] L. Fejér, Mechanische Quadraturen mit positiven Cotesschen Zahlen, Math. Z. 37 (1933), 287309.

[16] S. Filippi, Angenäherte Tschebyscheff-Approximation einer Stammfunktion-eine Modifikation des Verfahrens von Clenshaw und Curtis, Numer. Math. 6 (1964), 320-328.

[17] W. Fraser and M. W. Wilson. Remarks on the Clenshaw-Curtis quadrature scheme, SIAM Review 8 (1966), 322-327.

[18] P. V. Galkin, Estimates for the Lebesgue constants, Proc. Steklov Inst. Math. 109 (1971), 1-4.

[19] C. F. Gauss, Methodus nova integralium valores per approximationem inveniendi, Comment. Soc. Reg. Scient. Gotting. Recent., 1814 (also in Gauss's Werke, v. III, pp. 163-196).

[20] W. Gautschi, A survey of Gauss-Christoffel quadrature formulae, in P. L. Butzer and F. Fehér, eds., E. B. Christoffel, Birkhäuser, Basel, 1981, pp. 72-147.

[21] W. M. Gentleman. Implementing Clenshaw-Curtis quadrature I and II, Comm. ACM 15 (1972), 337-346, 353.

[22] G. H. Golub and J. H. Welsch, Calculation of Gauss quadrature rules, Math. Comp. 23 (1969), $221-230$.

[23] M. H. Gutknecht and L. N. Trefethen, Real polynomial Chebyshev approximation by the Carathéodory-Fejér method, SIAM J. Numer. Anal. 19 (1982), 358-371.

[24] J. P. Imhof, On the method for numerical integration of Clenshaw and Curtis, Numer. Math. 5 (1963), 138-141.

[25] D. K. Kahaner, Comparison of numerical quadrature formulas, in J. R. Rice, ed., Mathematical Software, Academic Press, New York, 1971.

[26] N. S. Kambo, Error bounds for the Clenshaw-Curtis quadrature formulas, BIT 11 (1971), 299309.

[27] Y. Katznelson, An Introduction to Harmonic Analysis, Dover, 1976.

[28] M. Kennedy and F. J. Smith, The evaluation of the JWKB phase shift, Mol. Phys. 13 (1967), 443-448. 
[29] A. R. Krommer and C. W. Ueberhuber, Computational Integration, SIAM, Philadelphia, 1998.

[30] V. I. Krylov, Approximate Calculation of Integrals, Macmillan, New York, 1962.

[31] J. C. Mason and D. C. Handscomb, Chebyshev Polynomials, Chapman and Hall/CRC, 2003.

[32] J. McNamee, Error-bounds for the evaluation of integrals by the Euler-Maclaurin formula and by Gauss-type formulae, Math. Comp. 18 (1964), 368-381.

[33] L. P. Natanson, Konstruktive Funktionentheorie, Akademie-Verlag, Berlin, 1955.

[34] S. E. Notaris, Interpolatory quadrature formulae with Chebyshev abscissae, J. Comp. Appl. Math. 133 (2001), 507-517.

[35] H. O'Hara and F. J. Smith, Error estimation in the Clenshaw-Curtis quadrature formula, Computer J. 11 (1968), 213-219.

[36] J. Ouspensky, Sur les valeurs asymptotiques des coefficients de Cotes, Bull. Amer. Math. Soc. 31 (1925), 145-156.

[37] G. Pólya, Über die Konvergenz von Quadraturverfahren, Math. Z. 37 (1933), 264-286.

[38] T. J. Rivlin, Chebyshev Polynomials: From Approximation Theory to Algebra and Number Theory, 2nd ed., Wiley, 1990.

[39] A. H. Stroud and D. Secrest, Gaussian Quadrature Formulas, Prentice-Hall, 1966.

[40] H. Takahasi and M. Mori, Estimation of errors in the numerical quadrature of analytic functions, Applicable Anal. 1 (1971), 201-229.

[41] L. N. Trefethen, Spectral Methods in MATLAB, SIAM, Philadelphia, 2000.

$[42]$ L. N. Trefethen, J. A. C. Weideman, and T. Schmelzer, Talbot quadratures and rational approximations, BIT, to appear.

[43] B. von Sydow, Error estimates for Gaussian quadrature formulae, Numer. Math. 29 (1977), $59-64$.

[44] J. Waldvogel, Fast construction of the Fejér and Clenshaw-Curtis quadrature rules, BIT 46 (2006), 195-202.

[45] J. A. C. Weideman, Explicit error formulas for interpolatory quadrature rules for rational integrands, manuscript, 2006.

[46] K. Wright, Series methods for integration, Computer J. 9 (1966), 191-199.

[47] W. P. Ziemer, Weakly Differentiable Functions, Springer, 1989. 